INPLASY

PROTOCOL

To cite: Wu et al. Can sexual intercourse or masturbation be a new therapy for distal ureteric stones: an updated meta-analysis. Inplasy protocol 202130066. doi:

10.37766/inplasy2021.3.0066

Received: 18 March 2021

Published: 19 March 2021

Corresponding author: Qiao Wu

2775310211@qq.com

Author Affiliation:

Affiliated Hospital of North of Sichuan Medical College

Support: 19SXHZ0185.

Review Stage at time of this submission: Data extraction.

Conflicts of interest:

None declared.

\section{Can sexual intercourse or masturbation be a new therapy for distal ureteric stones: an updated meta-analysis}

Wu, Q1; Liang, R²; Huang, Y3; Tan, C4; Tang, T5.

Review question / Objective: The P. I. C. O search tool was applied as follows: P: patients with distal ureteral stones; I: patients with distal ureteral stones treated with sexual intercourse or masturbation; C: patients with distal ureteral stones treated with tamsulosin or symptomatic therapy; 0 : stone expulsion rate at the second week and at the fourth week, number of needed analgesic injections, and stone expulsion time.

Condition being studied: A meta-analysis, which included three original studies, only involved male patients, and only compared sexual intercourse with placebo, concluded that intercourse was effective in the treatment of distal ureteral calculi. But a recent study has reported the efficacy of sexual intercourse in women with distal ureteral stones. There was also a study that reported the effects of sexual intercourse after extracorporeal shock wave lithotripsy for patients with distal ureteral calculi.Another study reported the efficacy of masturbation in the treatment of distal ureteral calculi.

INPLASY registration number: This protocol was registered with the International Platform of Registered Systematic Review and Meta-Analysis Protocols (INPLASY) on 19 March 2021 and was last updated on 19 March 2021 (registration number INPLASY202130066).

\section{INTRODUCTION}

Review question / Objective: The P. I. C. O search tool was applied as follows: P: patients with distal ureteral stones; I: patients with distal ureteral stones treated with sexual intercourse or masturbation; C: patients with distal ureteral stones treated with tamsulosin or symptomatic therapy; O: stone expulsion rate at the second week and at the fourth week, number of needed analgesic injections, and stone expulsion time.

Condition being studied: A meta-analysis, which included three original studies, only involved male patients, and only compared 
sexual intercourse with placebo, concluded that intercourse was effective in the treatment of distal ureteral calculi. But a recent study has reported the efficacy of sexual intercourse in women with distal ureteral stones. There was also a study that reported the effects of sexual intercourse after extracorporeal shock wave lithotripsy for patients with distal ureteral calculi.Another study reported the efficacy of masturbation in the treatment of distal ureteral calculi.

\section{METHODS}

Participant or population: Patients with distal ureteral stones.

Intervention: Sexual intercourse or masturbation.

Comparator: Tamsulosin therapy, symptomatic therapy.

\section{Study designs to be included: RCT.}

Eligibility criteria: Inclusion criteria: (1) studies that evaluated the effectiveness of sexual intercourse or masturbation for management of distal ureteral calculi, (2) studies that offered direct data or indirect data that could be calculated, including mean expulsion time of distal ureteric calculi, number of required analgesic injections, and expulsion rate of distal ureteric calculi, (3) studies that the full text was available.

Information sources: PubMed, EMBASE, and Cochrane Library.

Main outcome(s): Stone expulsion rate at the second week and at the fourth week, number of needed analgesic injections, and stone expulsion time.

Quality assessment / Risk of bias analysis: The quality of included RCTs was evaluated by using the revised Jadad Scale. The quality of each included study was determined by sequence generation, concealment of allocation, blinding method, number of cases lost to follow-up.
Strategy of data synthesis: RevMan version 5.3 was used to analyze the data. The mean difference (MD) for continuous variables was evaluated by Mantel-Haenszel method and DerSimonian and Laird method and the Odds Ratio (OR) was used to measure dichotomous variables results that pooled across studies, with corresponding 95\% confidence intervals $(\mathrm{Cls})$. If there is conspicuous heterogeneity, the random effects model is used; if there is no significant heterogeneity, the fixed effects model is used. Heterogeneity was assessed by the chi-squared tests. $P<0.05$ was considered to be significantly different.

Subgroup analysis: Due to the small number of original studies, no subgroup analysis was performed.

Sensitivity analysis: Due to the small number of original studies, no sensitivity analysis was performed.

Language: English.

Country(ies) involved: China, the US.

Keywords: Sexual intercourse; Masturbation; Distal ureteral stone; Heterogeneity; Meta-analysis.

Contributions of each author:

Author 1 - Qiao Wu.

Email: 2775310211@qq.com

Author 2 - Rui Liang.

Email: liangrui0416@163.com

Author 3 - Yi Huang.

Email: 1206634588@qq.com

Author 4 - Chunlin Tan.

Email: tanmed@outlook.com

Author 5 - Tielong Tang.

Email: cdzt2004@163.com 